\title{
Non-convulsive status epilepticus after cardiac surgery with cardiopulmonary bypass
}

\section{K. Skhirtladze-Dworschak', R. Jung², S. Aull Watschinger², E. Pataraia², M. Dworschak ${ }^{1}$}

\author{
1 Department of Anaesthesia, Intensive Care Medicine and Pain Medicine \\ Div. of Cardio-Thoracic and Vascular Anaesthesia and Intensive Care Medicine \\ 2 Department of Neurology, Medical University of Vienna, Vienna, Austria
}

Background and Goal of Study: Seizures after cardiac surgery (CS) have been charcterised as type II adverse neurologic outcome (1). They occur, on average in 5 - 70 of 1000 adult CS patients (2). In contrast to generalised tonic-clonic convulsions, detection of non-convulsive seizures in an intensive care environment is challenging as they can easily be confused with e.g. oversedation or confusion. A delay in the diagnosis, however, particularly of nonconvulsive status epilepticus, has been associated with a tremendous rise in morbidity and mortality (3). Furthermore, due to the difficulties related to the recognition of non-convulsive status epilepticus, its incidence after cardiac surgery is still unknown.

Materials and Methods: We, therefore, retrospectively assessed electroencephalograms (EEG) of patients who had undergone either isolated coronary artery bypass graft (CABG) surgery or aortic valve replacement (AVR) both with cardiopulmonary bypass between January 1, 2014 and June 30, 2015. All EEGs had been ordered by a consultant neurologist to either confirm or rule out suspected perioperative brain injury and were reanalysed by three epileptologists.

Fig.

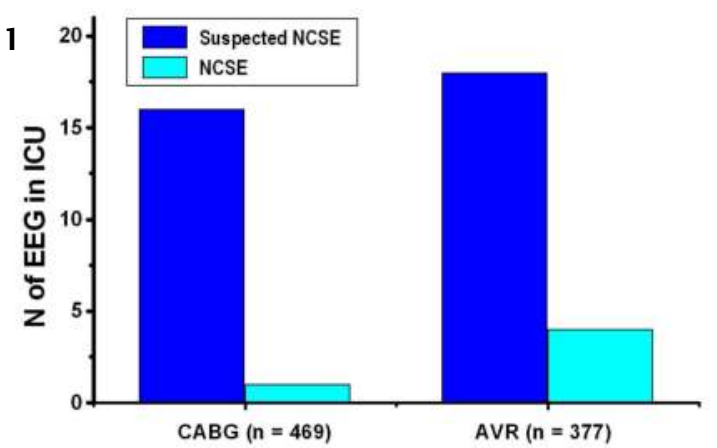

Conclusion: Patients after open heart surgery with unexplained prolonged unresponsiveness require special attention. EEG recordings should probably be ordered more liberally to allow early detection of this elusive and potentially devastating type of neuronal hyperexcitation.

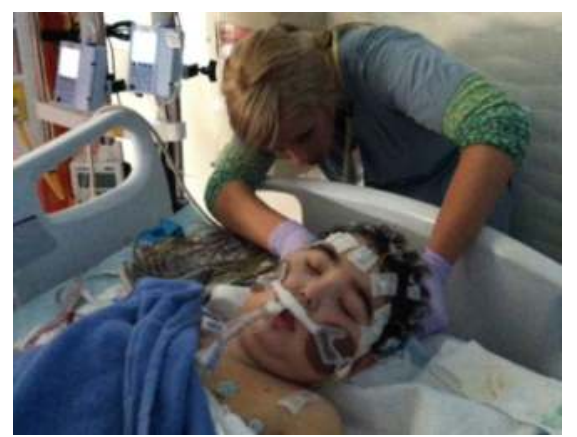

Non-convulsive seizure in an ICU setting

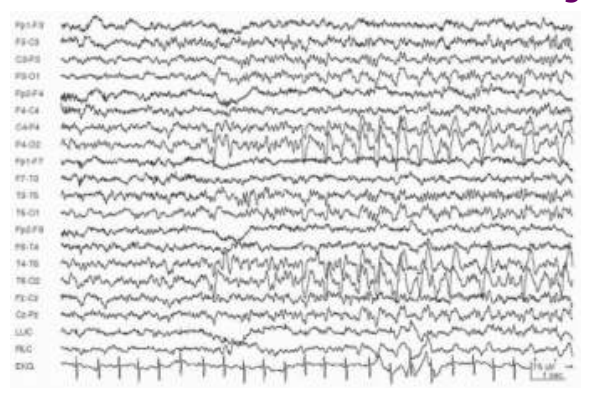

Results and Discussion: During the above mentioned period a total of 469 isolated CABG and 377 isolated AVR procedures were carried out. At the same time 18 EEGs were recorded in AVR patients and 16 in isolated CABG patients during their stay in the intensive care unit. Non-convulsive status epilepticus could be detected in 4 AVR patients $(0.9 \%)$ and in 1 patient $(0.3 \%)$ after CABG surgery (Fig. 1). Despite the limitations of this trial, particularly its retrospective design and the individual decision of neurology consultants to order an EEG, it appears that it is not only the incidence of seizures that increases with open heart surgery but also the incidence of nonconvulsive status epilepticus. The threefold greater incidence of this frequently missed seizure type makes patients undergoing open heart surgery more vulnerable for more severe adverse postoperative cerebral outcome.

References: 1) Roach GW et al. N Engl J Med 1996;335:1 857-63. 2) Sharma V et al. Anaesthesia 2014;69:124-30. 3) Young GB et al. Neurology 1996;47:83-9 https://intensivecarehotline.com/electroencephalograph-eeg/ https://neupsykey.com/nonconvulsive-status-epilepticus/ 